\title{
The co-orbital restricted three-body problem and its application
}

\author{
YI ZhaoHua $^{1,3}$, LI GuangYu ${ }^{1 *}$, Gerhard HEINZEL ${ }^{2}$, Albrecht RÜDIGER ${ }^{2}$, LUO YongJie ${ }^{1}$, XIA \\ Yan $^{1} \&$ ZHAO HaiBin ${ }^{1}$ \\ ${ }^{1}$ Purple Mountain Observatory, Chinese Academy of Sciences, Nanjing 210008, China; \\ ${ }^{2}$ Max Planck Institute for Gravitational Physics, D-30167 Hannover, Germany; \\ ${ }^{3}$ Department of Astronomy, Nanjing University, Nanjing 210008, China
}

Received July 3, 2009; accepted November 27, 2009

\begin{abstract}
Based on large quantities of co-orbital phenomena in the motion of natural bodies and spacecraft, a model of the co-orbital restricted three-body problem is put forward. The fundamental results for the planar co-orbital circular restricted three-body problem are given, which include the selection of variables and equations of motion, a set of approximation formulas, and an approximate semi-analytical solution. They are applied to the motion of the barycenter of the planned gravitational observatory LISA constellation, which agrees very well with the solution of precise numerical integration.
\end{abstract}

co-orbital, restricted three-body problem, LISA

The co-orbital phenomena in the motion of natural bodies were paid attention to by astronomers a long time ago. In the year 1918 the Japanese astronomer Hirayama found the orbital elements of some asteroids close to each other (especially semi-major axis $a$, eccentricity $e$, and orbital inclination $i$, the ones with a close $a$ were later called an asteroid group. He proposed that asteroids with at least two out of $a, e, i$, close to each other be called an asteroid family, and that with all three elements close to each other be called co-orbital, which is discussed in this paper. To discuss the problem of common origin, he also proposed the concept of proper elements [1]. As the number of minor planets having been found is increasing rapidly in recent years, the study of asteroid families has greatly developed. Many methods of the definition and calculation of proper orbital elements have been put forward, and the deeper understanding of many aspects, which includes the statistics, dynamics, and spectral types, of asteroid sub-families, has been brought forth. And a relatively comprehensive summary was given by Lemaitre [2] in 2005.

*Corresponding author (email: gyl@pmo.ac.cn)

Recommended by ZHOU JiLin
The most well-known cases of co-orbits in natural objects are Trojan groups of asteroids, of which the number of the registered asteroids already found has exceeded 2900. There also exist co-orbital phenomena both in the motion of stars in the same spiral arm of the Milky Way galaxy, and in the motion of natural and man-made satellites. The planned gravitational wave observatory LISA is allowed to be regarded as approximately co-orbital, of which the barycenter of the three spacecraft co-orbits with the earth and the distance between each spacecraft and the barycenter is very small (less than $0.02 \mathrm{AU}$ ). We have taken part in the LISA orbit design and optimization; since the required operating period is merely 10 years, we have simply made use of a rough method $[3,4]$ to deal with it, and obtained relatively ideal results.

Although the classical restricted three-body problem has already had a wide range of applications, it has not been fully resolved. In recent years, lots of researches into co-orbital motion appear, which are mainly aimed at the quasi-satellite (QS for short) case, such as the work by Mikkola et al. [5]. On the basis of widespread co-orbital phenomena in the motion of natural and man-made celestial objects we put forward the model of co-orbital restricted 
three-body problem, of which further results are expected to be obtained and to be applied to more objects. In this paper, the most fundamental planar co-orbital circular restricted three-body problem is discussed first. In sec. 1-3 a discussion of the selection of variables and equations of motion, an important approximation formula, and the approximate analytical method are presented; in Sec. 5 the motion of the barycenter of the LISA constellation is taken as an example, of which the results are compared with those of a precise numerical method.

\section{Selection of variables and equations of motion}

Take the solar system for an example. Assume $\mathrm{S}$ and $\mathrm{E}$ to represent the sun and a certain major planet respectively. Assume the planet $\mathrm{E}$ to move round the sun $\mathrm{S}$ along a circular orbit, whose orbital angular velocity is $n$, being a constant. Set $\mathrm{S}$ as the origin of coordinates, and SE as the direction of the $x$ axis, and then construct a rotating coordinate system (synodic coordinate system) which rotates round $\mathrm{S}$ with E. For the sake of convenience, assume the masses of $\mathrm{S}$ and $\mathrm{E}$ to be $1-\mu$ and $\mu$ respectively, and the distance of SE to be unit of length. The coordinates $(x, y)$ of the minor object $\mathrm{P}$ on the plane should satisfy the equations [3] below:

$$
\ddot{x}-2 n \dot{y}=\frac{\partial \Omega}{\partial x}, \quad \ddot{y}+2 n \dot{x}=\frac{\partial \Omega}{\partial y} ; \quad \Omega=\Omega_{0}+\mu \Omega_{1},
$$

in which

$$
\begin{aligned}
& \Omega=\frac{n^{2}}{2}\left[(1-\mu) r^{2}+\mu \Delta^{2}\right]+n^{2}\left(\frac{1-\mu}{r}+\frac{\mu}{\Delta}\right), \\
& \Omega_{0}=n^{2}\left(\frac{r^{2}}{2}+\frac{1}{r}\right), \quad \Omega_{1}=n^{2}\left(\frac{1}{2}-x-\frac{1}{r}+\frac{1}{\Delta}\right), \\
& r^{2}=x^{2}+y^{2}, \quad \Delta^{2}=r^{2}-2 x+1 .
\end{aligned}
$$

To facilitate calculation, the dimensionless unit system is not fully adopted, and the coordinate rotation angular velocity $n$ is retained in the equations.

Now transform the rectangular coordinates into polar coordinates $r, \vartheta$, i.e.

$$
x=r \cos \vartheta, \quad y=r \sin \vartheta .
$$

Substitute eq. (3) into eq. (1) and convert it to equations of motion with $r, \vartheta$ as variables:

$$
\begin{aligned}
& \ddot{r}-r \dot{\vartheta}^{2}-2 n r \dot{\vartheta}=\frac{\partial \Omega}{\partial r} \\
& =n^{2} r-\frac{n^{2}}{r^{2}}+\mu n^{2}\left(\frac{1}{r^{2}}-\cos \vartheta-\frac{r-\cos \vartheta}{\Delta^{3}}\right), \\
& \frac{\mathrm{d}}{\mathrm{d} t}\left[r^{2}(\dot{\vartheta}+n)\right]=\frac{\partial \Omega}{\partial \vartheta}=\mu n^{2} r \sin \vartheta\left(1-\frac{1}{\Delta^{3}}\right) . \\
& \Delta^{2}=r^{2}-2 r \cos \vartheta+1 .
\end{aligned}
$$

Eq. (4) has a Jacobian integral:

$$
\dot{r}^{2}+r^{2} \dot{\vartheta}^{2}=2 \Omega-n^{2} C,
$$

in which $C$ is known as the Jacobian integration constant.

In the above equations, $\mu$ approximates the ratio of the planet's mass to the sun's, which is a small quantity less than 0.001 in the solar system. We first discuss the case $\mu=0$, namely the restricted two-body problem, or the two-body problem in a rotating coordinate system. Taking

$$
\psi=\vartheta+n t, \quad \text { or } \quad \dot{\psi}=\dot{\vartheta}+n,
$$

eq. (4) is converted to equations of motion of the two-body problem; it can be worked out following the method for the two-body problem:

$$
r^{2} \dot{\psi}=h ; \quad r=\frac{h^{2} / n^{2}}{1+e \cos (\psi+B)} ; \quad \dot{r}=\frac{e n^{2}}{h} \sin (\psi+B) .
$$

There is one more integral containing another integration constant, which is to be solved necessarily according to the specific conical sections. As a consequence, the integration constants for the restricted two-body problem are allowed to be chosen as $h, e, B$, with the other constant is neglected.

Based on the restricted two-body problem, using the method of the variation of arbitrary constants, when $\mu \neq 0$, the equations of motion are derived to be

$$
\begin{gathered}
\dot{h}=\mu n^{2} r \sin \vartheta\left(1-\frac{1}{\Delta^{3}}\right), \\
\dot{e}=\mu h K \sin (\psi+B)+\frac{\dot{h}}{h}\left[e+\left(1+\frac{h^{2}}{n^{2} r}\right) \cos (\psi+B)\right], \\
e \dot{B}=\mu h K \cos (\psi+B)-\left(1+\frac{h^{2}}{n^{2} r}\right) \sin (\psi+B),
\end{gathered}
$$

and from eqs. (6), (7) we get

$$
\dot{\vartheta}=\dot{\psi}-n=\frac{h}{r^{2}}-n,
$$

where

$$
K=\frac{1}{r^{2}}-\cos \vartheta-\frac{r-\cos \vartheta}{\Delta^{3}}, \quad r=\frac{h^{2} / n^{2}}{1+e \cos (\psi+B)} .
$$

Eqs. (8)-(11) are equations of motion of the planar circular restricted three-body problem, in the perturbed two-body form. To avoid the difficulty of small eccentricity, we introduce $u$ and $v$ to substitute for $e$ and $B$ :

$$
u=e \cos (\psi+B), \quad v=e \sin (\psi+B),
$$

we can derive that: 


$$
\dot{u}=\frac{2 \dot{h}}{h}(1+u)-\frac{h}{r^{2}} v, \quad \dot{v}=\mu h K+\frac{\dot{h}}{h} v+\frac{h}{r^{2}} u,
$$

where

$$
r=\frac{h^{2} / n^{2}}{1+u}
$$

Thus eqs. (8), (11) and eq. (14) together make up the equations of motion of the planar circular restricted three-body problem, in which $r$ should be substituted by eq. (15). The principal variables become $h, \vartheta, u, v$.

\section{The planar co-orbital circular restricted three-body problem}

An arbitrary point on the circumference of a unit circle with the Sun being its center

$$
r=1, \quad \vartheta=\vartheta_{0} \quad(\text { arbitrary constant })
$$

is a particular solution and a Lagrange libration point as well, at which celestial bodies situated are all co-orbital with the planet. The co-orbital restricted three-body problem proposed in this paper studies the particular law of motion of the minor object whose initial position is given by eq. (16).

Substituting the initial conditions (16) into the Jacobian integral (5) yields

$$
C=3(1-\mu)+\mu\left(4 \sin ^{2} \frac{\vartheta_{0}}{2}+\csc \frac{\vartheta_{0}}{2}\right)
$$

This is the Jacobian constant of the co-orbital restricted three-body problem, depending only on $\vartheta_{0}$ and $\mu$. The curve given in Figure 1 illustrates the relation between $C$ and $\vartheta_{0}$ when $\mu=3.0359 \times 10^{-6}$ (system of Sun and Earth-Moon):

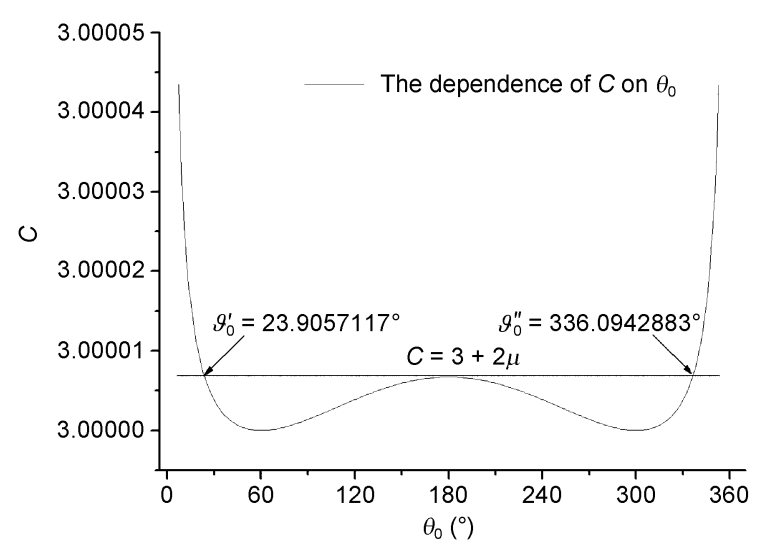

Figure 1 The relation between $C$ and $\vartheta_{0}$ for the system of Sun and Earth-Moon.
When $\vartheta_{0}=60^{\circ}$ and $300^{\circ}$ (corresponding to the libration points $L_{4}$ and $L_{5}$ ), $C$ takes a minimum value of 3 , independent of $\mu$. When $\vartheta_{0}=180^{\circ}, C=3+2 \mu$, being a maximum. The line $C=3+2 \mu$ intersects the curve $C$ at two other points $\vartheta_{0}^{\prime}=23^{\circ} .9057117$ and $\vartheta_{0}^{\prime \prime}=336^{\circ} .0942883$, neither being dependent on $\mu$.

The initial conditions (16) indicate that the minor object is situated on the zero-velocity curve at the initial moment. The curve serves as the boundary between the permissible area and prohibited region of the minor object. Out of the zero-velocity curves (the corresponding Jacobian constants are $C_{i}$, when sorting by magnitude $C_{1}$ is the biggest) those that pass the libration points $L_{i}(i=1,2,3,4,5)$ are of particular importance; their intersecting points with a unit circle are boundary points, and the corresponding arguments $\vartheta_{0}$ equal to $\vartheta_{0 i}$ for them each. Table 1 lists the values of the arguments corresponding to the boundary points for the two cases: $\mu=3.0359 \times 10^{-6}$ (system of Sun and EarthMoon) and $\mu=0.9538754 \times 10^{-3}$ (system of Sun and Jupiter).

Because of symmetry, the points with $r=1$ and $\vartheta=-\vartheta_{0 i}$ are also on the curve of zero velocity of $C=C_{i}$. Therefore the plane of motion is divided into different regions by the curves of zero velocity corresponding to values of each $\vartheta_{0 i}$, and the relevant motion is confined to a certain region.

As is shown in Figure 2, when the absolute value of $\vartheta_{0}$ is less than $\vartheta_{01}$, the motion is confined to an approximately circular region in the neighborhood of the planet, called the quasi-satellite region (QS for short); when the absolute value of $\vartheta_{0}$ is between $\vartheta_{01}$ and $\vartheta_{02}$, the motion is confined to the interior of a dumbbell-shaped region, which is called the dumbbell region (DB); when the absolute value of $\vartheta_{0}$ is between $\vartheta_{02}$ and $\vartheta_{03}$, the prohibited region is the interior of a horse-shoe-shaped region, the motion is confined to the exterior of the horse-shoe-shaped region, called horse-shoe region (HS); when the absolute value of $\vartheta_{0}$ is larger than $\vartheta_{03}$, the prohibited region shrinks to the interior of two symmetric tadpole-shaped regions, the curves of motion are confined to the exterior of the tadpole-shaped regions, which is called the tadpole region (TP).

$\vartheta_{03}$ is slightly different from $\vartheta_{0}^{\prime}$, and the corresponding

Table 1 Values of different $\vartheta_{0}$ for the system of Sun and Earth-Moon, and the system of Sun and Jupiter

\begin{tabular}{cccccc}
\hline \multicolumn{1}{c}{$\mu$} & $\vartheta_{01}$ & $\vartheta_{02}$ & $\vartheta_{03}$ & $\vartheta_{04}$ & $\vartheta_{05}$ \\
\hline $3.0359 \times 10^{-6}$ & $0^{\circ} .3828225$ & $0^{\circ} .3845339$ & $23^{\circ} .9056$ & $60^{\circ}$ & $300^{\circ}$ \\
$0.9538754 \times 10^{-3}$ & $2^{\circ} .5675658$ & $2^{\circ} .64665725$ & $23^{\circ} .9069$ & $60^{\circ}$ & $300^{\circ}$ \\
\hline
\end{tabular}




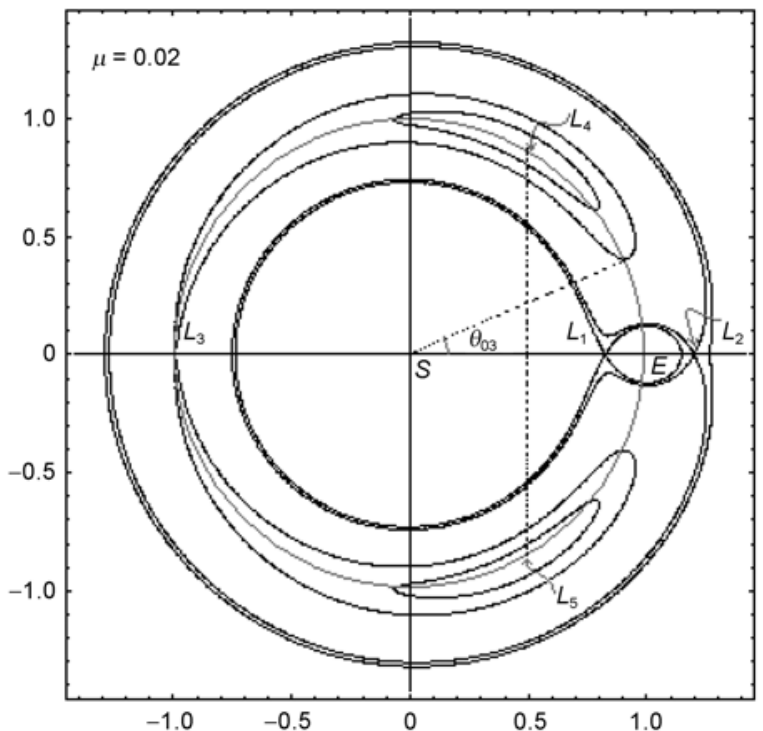

Figure 2 Diagram of several permissive areas (to facilitate drawing, $\mu=$ 0.02 was taken).

Jacobian constant $C_{3}<3+2 \mu$. When $\mu$ is very small (in the case of the system of Sun and a planet), the difference can be neglected in an approximate discussion. However, owing to singularity, when met with the case $\vartheta_{0} \approx \vartheta_{0 i}$ in an application, a stricter method should be used.

\section{The approximate analytical solution}

Though starting from the initial co-orbital conditions, the orbits corresponding to arbitrary values of $\vartheta_{0}$ can be precisely computed with a numerical solution, nevertheless, an analytical solution, even though only approximate, is of much significance, for the sake of discussing properties of orbits within different regions of motion to facilitate their application.

\subsection{Parameter $\tau$ and its approximation formula}

For the case of $\mu$ being very small such as the solar system, an analytical solution accurate to the first order of $\mu$ can be developed. The initial value of the eccentricity $e$ is zero, $\dot{e}$ contains the factor of $\mu$; so $e, u$ and $v$ are all small quantities of the first order, and among them $u, v$ are short-period terms. They can be discussed separately. First, assume $u, v$ to be zero, from eqs. (15), (8), (11) we get

$$
r=\frac{h^{2}}{n^{2}}, \quad \dot{h}=\mu r n^{2} \sin \vartheta\left(1-\frac{1}{\Delta^{3}}\right), \quad \dot{\vartheta}=\frac{h}{r^{2}}-n .
$$

They can be converted to

$$
\dot{h}=\mu h^{2} \sin \vartheta\left(1-\frac{1}{\Delta^{3}}\right), \quad \dot{\vartheta}=\frac{n^{4}}{h^{3}}-n .
$$

With the accuracy of first-order perturbation of $\mu$ and adopting $r=1$ in the expression of $\Delta$, yields $\Delta=\sin (\vartheta / 2)>$ 0 (because $\left.\vartheta \in\left(0^{\circ}, 360^{\circ}\right), \sin (\vartheta / 2)>0\right)$. Again let

$$
h=n(1+\tau)
$$

to introduce the significant parameter $\tau$ in our discussion, the above equation is converted to

$$
\left[(1+\tau)^{-5}-(1+\tau)^{-2}\right] \mathrm{d} \tau=\mu \sin \vartheta\left(1-\frac{1}{8} \sin ^{-3} \frac{\vartheta}{2}\right) \mathrm{d} \vartheta
$$

Integrating it, we get

$$
\begin{aligned}
& -\frac{3}{4}-\frac{1}{4} \frac{1}{(1+\tau)^{4}}+\frac{1}{1+\tau} \\
& =\mu\left[\left(\cos \vartheta_{0}-\cos \vartheta\right)+\frac{1}{2}\left(\csc \frac{\vartheta}{2}-\csc \frac{\vartheta_{0}}{2}\right)\right]
\end{aligned}
$$

Expanding the left-hand side with respect to $\tau$, we obtain $-\frac{3}{2} \tau^{2}+4 \tau^{3}+\cdots$, if it only needs to be accurate to $\tau^{2}$, we have

$$
\tau^{2}=\frac{2}{3} \mu Q(\vartheta)
$$

where

$$
Q(\vartheta)=Y(\vartheta)-Y\left(\vartheta_{0}\right), \quad Y(\vartheta)=\cos \vartheta-\frac{1}{2} \csc \frac{\vartheta}{2} .
$$

From this we can see that $\tau$ is the same magnitude of $\sqrt{\mu}$, when $\mu$ is a small quantity. In Figure 3 the relation between $\tau^{2}$ and $\vartheta$ for $\vartheta_{0}=20^{\circ}$ is presented.

From eq. (20), we know that the motion has the following properties:

a) being non-negative: $Q(\vartheta) \geqslant 0$;

b) symmetry: $Q(-\vartheta)=Q\left(360^{\circ}-\vartheta\right)=Q(\vartheta)$; accordingly it is only necessary to discuss the case $0^{\circ} \leqslant \vartheta_{0} \leqslant 180^{\circ}$;

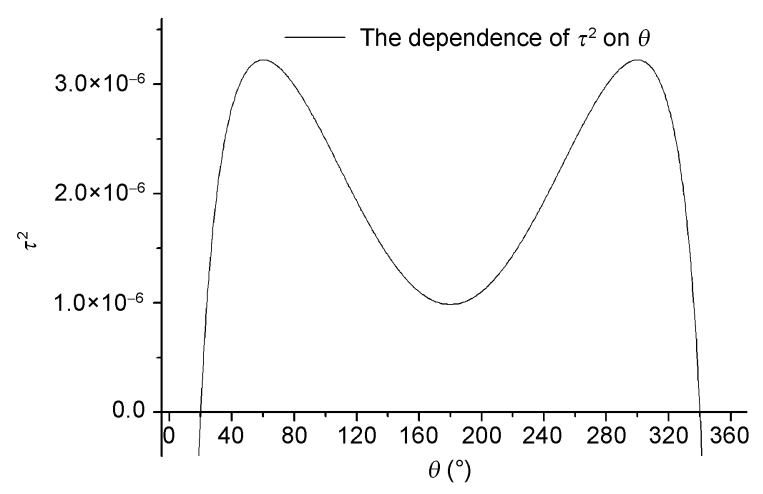

Figure 3 Relation between $\tau^{2}$ and $\vartheta$ for $\vartheta_{0}=20^{\circ}$. 
c) when $\vartheta_{02}<\vartheta_{0}<\vartheta_{03}$, i.e., in the HS region, the range within which $\vartheta$ varies is $\left(\vartheta_{0}, 360^{\circ}-\vartheta_{0}\right)$;

d) when $\vartheta_{0}>\vartheta_{03}$, i.e., in the TP region, the range within which $\vartheta$ varies is $\left(\vartheta_{0}, \vartheta_{1}\right)$, where $\vartheta_{1}$ is determined by the following equation:

$$
\sin \frac{\vartheta_{1}}{2}=-\frac{1}{2} \sin \frac{\vartheta_{0}}{2}+\frac{1}{2} \sqrt{\sin ^{2} \frac{\vartheta_{0}}{2}+\csc \frac{\vartheta_{0}}{2}}
$$

For the case of $\vartheta_{0}<\vartheta_{02}$, another discussion is necessary.

\subsection{The approximate solution neglecting the short- period term}

Now aiming at the two cases of HS and TP regions, following the above results, we develop an approximate analytical solution accurate to the firs-order of $\mu$. Since the initial value of the eccentricity $e$ is zero, the rate of variation $\dot{e}$ being a small quantity of the first order, as a general rule, the eccentricity should be a first-order small quantity. And then,

$$
u=e \cos (\psi+B)=e \cos (n t+\vartheta+B)
$$

is a short-period term of the first order. Therefore in the analytical solution of the first order, it can be solved separately, then combined with other terms. First we neglect $u$ and work at other terms, the corresponding equations are

$$
\begin{aligned}
& r=\frac{h^{2}}{n^{2}}=(1+\tau)^{2}, \quad \tau^{2}=\frac{2}{3} \mu Q(\vartheta), \\
& \dot{\vartheta}=n\left[\frac{1}{(1+\tau)^{3}}-1\right]=n\left(-3 \tau+6 \tau^{2}-\cdots\right) .
\end{aligned}
$$

From these we get

$$
\tau= \pm \sqrt{\frac{2}{3} \mu Q(\vartheta)}
$$

in which the plus and minus signs are determined by $\vartheta_{0}$ according to the relation

$$
\dot{\tau}=\frac{\dot{h}}{n}=\mu n \sin \vartheta_{0}\left(1-\frac{1}{8} \csc ^{3} \frac{\vartheta_{0}}{2}\right) .
$$

For example, in the case of the barycenter of LISA spacecraft, $\dot{\tau}>0$ when $\vartheta_{0}=-20^{\circ}$ or $340^{\circ}$, whereas $\tau=0$ when $t=0$. Accordingly when $t>0$ (at least in the neighborhood), we have

$$
\tau>0, \dot{\vartheta}<0
$$

In a similar way, when $\vartheta_{0}=20^{\circ}$, correspondingly we get $\tau<0$ and $\dot{\vartheta}>0$.
In addition, following the properties of $Q(\vartheta)$ (see Figure 3), one can see that except $Q\left(20^{\circ}\right)=Q\left(340^{\circ}\right)=0$, $Q(\vartheta)$ has no more zero points in the range $20^{\circ}<\vartheta<340^{\circ}$ is allowed to maintain $\tau>0$ and $\dot{\vartheta}<0$, therefore $\vartheta$ decreases monotonically from $340^{\circ}$ to $20^{\circ}$, coming back to $\tau=0 \quad(r=1)$; similarly, again taking $\vartheta_{0}=20^{\circ}$ as a new starting point, meanwhile $\dot{\tau}<0$, as the time increases one gets $\tau<0$ and $\dot{\vartheta}>0$; consequently $\vartheta$ increases monotonically from $20^{\circ}$ to $340^{\circ}$. The process can keep repeating itself. Hence, the solution developed this way is a periodic solution, it is only necessary to give the formula for calculation in one period. Owing to the aforementioned characteristics, the formula needs to be divided into two parts to calculate. For the case of the barycenter of LISA spacecraft, starting from $340^{\circ}$ we have

$$
\begin{aligned}
n t & =\int_{340^{\circ}}^{\vartheta} \frac{\mathrm{d} \vartheta}{-\sqrt{6 \mu Q(\vartheta)}+4 \mu Q(\vartheta)} \\
& =\int_{\vartheta}^{340^{\circ}} \frac{\mathrm{d} \vartheta}{\sqrt{6 \mu Q(\vartheta)}-4 \mu Q(\vartheta)},
\end{aligned}
$$

and starting from $20^{\circ}$ we have

$$
n t=\int_{20^{\circ}}^{\vartheta} \frac{\mathrm{d} \vartheta}{\sqrt{6 \mu Q(\vartheta)}+4 \mu Q(\vartheta)} .
$$

The function $Q(\vartheta)$ is defined by eq. (20), the above mentioned integration cannot be expressed by elementary functions, and it can only be computed numerically, thus obtaining the functional relation between the argument $\vartheta$ and the time $t$. Using $T_{1}$ and $T_{2}$ to represent the time (take a year as the time unit) it takes when $\vartheta$ decreases from $340^{\circ}$ to $20^{\circ}$, and increases from $20^{\circ}$ to $340^{\circ}$, respectively, we have

$$
\begin{aligned}
& T_{1}=\frac{1}{2 \pi} \int_{20^{\circ}}^{340^{\circ}} \frac{\mathrm{d} \vartheta}{\sqrt{6 \mu Q(\vartheta)}-4 \mu Q(\vartheta)}, \\
& T_{2}=\frac{1}{2 \pi} \int_{20^{\circ}}^{340^{\circ}} \frac{\mathrm{d} \vartheta}{\sqrt{6 \mu Q(\vartheta)}+4 \mu Q(\vartheta)} .
\end{aligned}
$$

The full period is then

$$
T=T_{1}+T_{2} \approx \frac{1}{\pi} \int_{20^{\circ}}^{340^{\circ}} \frac{\mathrm{d} \vartheta}{\sqrt{6 \mu Q(\vartheta)}} .
$$

The above mentioned results can be used directly in the case of the general HS region, for arbitrary $\vartheta_{02}<\vartheta_{0}<\vartheta_{03}$, eq. (28) becomes

$$
n t=\int_{\vartheta}^{\vartheta} \frac{\mathrm{d} \vartheta}{\sqrt{6 \mu Q(\vartheta)}+4 \mu Q(\vartheta)},
$$


and eq. (27)

$$
n t=\int_{\vartheta}^{2 \pi-\vartheta_{0}} \frac{\mathrm{d} \vartheta}{\sqrt{6 \mu Q(\vartheta)}-4 \mu Q(\vartheta)} .
$$

Meanwhile in expressions (29) and (30) of period $T$, it is only necessary to change the upper limit of the integration to $2 \pi-\vartheta_{0}$, and the lower limit to $\vartheta_{0}$.

In a similar way, for the case of $\mathrm{TP}$ region, when $60^{\circ}>\vartheta_{0}>\vartheta_{03}$, first calculate $\vartheta_{1}$ following eq. (21); it is easy to decide that $180^{\circ}>\vartheta_{1}>60^{\circ}$, $\vartheta$ can only vary between $\vartheta_{0}$ and $\vartheta_{1}$. The form of the corresponding eq. (31) does not change, but in eq. (32) the upper limit of the integration changes to $\vartheta_{1}$. In the expression (30) of the period $T$, it is necessary to change the upper limit of the integration to $\vartheta_{1}$, and the lower limit to $\vartheta_{0}$. When $180^{\circ}>\vartheta_{0}>60^{\circ}$, the corresponding $\vartheta_{1}$ should satisfy $60^{\circ}>\vartheta_{1}>\vartheta_{03}$; like above, but it is necessary to exchange the positions of $\vartheta_{0}$ and $\vartheta_{1}$. When $\vartheta_{0}>180^{\circ}$, we can make use of the relation of symmetry $Q\left(360^{\circ}-\vartheta\right)=Q(\vartheta)$ to directly use the results before.

\subsection{The short-period term}

In the first-order theory, the first-order short-period terms can be dealt with approximately. In the expressions of $t$ and $\vartheta$, the variable embodying the short-period term is the parameter $u$, below we derive its approximate expression. When $t=0, e=0, r=1$ and $h=n$, hence after simplification, we get

$$
\dot{e}=\mu n(K \sin f+2 L \cos f),
$$

in which

$$
\begin{aligned}
& f=\psi+B, \\
& K=\left(1-\cos \vartheta_{0}\right)\left(1-\frac{1}{8} \csc ^{3} \frac{\vartheta_{0}}{2}\right), \\
& L=\sin \vartheta_{0}\left(1-\frac{1}{8} \csc ^{3} \frac{\vartheta_{0}}{2}\right) .
\end{aligned}
$$

$K, L$ are constants, so we have

$$
\begin{aligned}
& \dot{e}=\alpha \sin (f+\gamma), \\
& \alpha=\sqrt{(\mu n K)^{2}+(2 \mu n L)^{2}}, \\
& \cos \gamma=\mu n K / \alpha, \quad \sin \gamma=2 \mu n L / \alpha .
\end{aligned}
$$

$\alpha$ is a first-order small quantity, we can adopt approximately $\dot{B}=0, \quad \dot{f}=\dot{\psi}+\dot{B}=h / r^{2}=n$, the above equation is converted to

$$
\frac{\mathrm{d} e}{\mathrm{~d} f}=\frac{\alpha}{n} \sin (f+\gamma)=\mu \sqrt{K^{2}+4 L^{2}} \sin (f+\gamma),
$$

the initial value of $e$ is 0 , so we integrate it and get

$$
e=\mu \sqrt{K^{2}+4 L^{2}}\left[\cos \left(f_{0}+\gamma\right)-\cos (f+\gamma)\right] .
$$

While $f=\psi+B=\vartheta+n t+B$ can take any arbitrary value as the time varies, to maintain $e \geqslant 0$, it requires $\cos \left(f_{0}+\gamma\right)=1$, namely $f_{0}=\vartheta_{0}+B=-\gamma$, as a result the expressions of $e$ and $u$ are

$$
\begin{aligned}
& e=\mu \sqrt{K^{2}+4 L^{2}}\left[1-\cos \left(\vartheta-\vartheta_{0}+n t\right)\right], \\
& u=e \cos \left(\vartheta-\vartheta_{0}+n t-\gamma\right),
\end{aligned}
$$

in which $\vartheta$ can be calculated through the given time $t$ following eq. (31) or (32). Substituting the the value of $\vartheta$ into eqs. (24) and (36) yields the values of the corresponding $\tau$ and $u$, therefore $r$ which contains the short-period terms can be calculated through the exact formula

$$
r=(1+\tau)^{2} /(1+u)
$$

As for the short-period term contained in $\vartheta$, it can be obtained with the more accurate expression

$$
\begin{aligned}
\dot{\vartheta} & =\frac{h}{r^{2}}-n=n\left[\frac{(1+u)^{2}}{(1+\tau)^{3}}-1\right] \\
& \approx n\left(-3 \tau+6 \tau^{2}\right)+2 n u=\dot{\vartheta}_{u=0}+\dot{\delta},
\end{aligned}
$$

where $\vartheta_{u=0}$ is the value of $\vartheta$ when $u=0$, discussed before. The additive short-period term is

$$
\delta=\int_{0}^{t} 2 n u \mathrm{~d} t=2 n \int_{0}^{t} e \cos \left(\vartheta-\vartheta_{0}+n t-\gamma\right) \mathrm{d} t,
$$

it can only be calculated numerically. When necessary, relationship $\vartheta=\vartheta_{u=0}+\delta$ can be used to iterate.

The practical calculation indicates that the rate of change of $\vartheta$ is almost constant, assumed to be $\bar{n}$. Under the condition that the accuracy is not be affected, we can adopt $\bar{n}=4\left(\pi-\vartheta_{0}\right) / T$ and $\bar{n}=2\left|\vartheta_{1}-\vartheta_{0}\right| / T$ for the HS and $\mathrm{TP}$ regions respectively, and we take

$$
\vartheta= \pm \bar{n} t+\vartheta_{0}
$$

in which the plus sign is taken for the segment in which $\vartheta$ increases, and the minus sign, for the segment in which $\vartheta$ decreases. Substituting the expression of $e$ in eq. (36) into eq. (39), we can get the analytical expression of $\delta$ through integration.

\section{Numerical check}

The solution presented above is a semi-analytical one consisting of a periodic-term plus a short-periodic term. As the 
integration cannot be expressed with elementary functions, it has to be computed with a numerical method. In this paper we made a discussion mainly aiming at two cases- the HS and TP regions, which already cover most of the range of the initial values for the motion of celestial bodies in the solar system. In the numerical check below, we find a very satisfactory agreement between the results gained by making integration over the equations of motion and those of the calculation using the above mentioned semi-analytical method.

\subsection{The motion of the barycenter of the LISA spacecraft}

The analysis of the motion of the barycenter of the LISA constellation is a typical application of the planar co-orbital restricted three-body problem. We adopt $r=1, \quad \dot{r}=\dot{\vartheta}=0$, $\vartheta_{0}=340^{\circ}$ when $t=0$, then make an precise numerical integration following the original equations (4) of motion in polar coordinates. It takes 85614.5 days or 234.3997 years for $\vartheta$ to monotonically decrease from $340^{\circ}$ to $20^{\circ}$ (there will be minute short-period disturbances), during which $r>1$ and there are two minimum and one maximum values in the variation. As the computation continues, it takes another 85343.51145 days or 233.6578 years for $\vartheta$ to monotonically increase from $20^{\circ}$ to $340^{\circ}$, during which $r<1$ and there are two maximum and one minimum values. The curve of zero velocity corresponding to that initial condition is horse-shoe-shaped. The computation results indicate that the barycenter of LISA starts out from $r=1$, $\vartheta=\vartheta_{0}=340^{\circ}$, moves in the neighborhood along the outer circumference of the horse-shoe-shape, after arriving at $\vartheta=20^{\circ}$, it then returns to $\vartheta=340^{\circ}$ along the neighboring inner circumference of the horse-shoe-shaped, which accords with the results of the semi-analytical method in this paper. For details of comparison please refer to the following figures. Figures 4(a) and 4(b) are comparisons of the computation results of $r$ and $\vartheta$ which are obtained by numerical integration with those obtained by the semi-analytical solution in this paper.

Following eq. (29), $T_{1}$ and $T_{2}$ are calculated to be as follows:

$$
T_{1}=234.66 \text { years, } T_{2}=233.47 \text { years, }
$$

which approximates the results by numerical integration.

Here we have calculated the relation between the period $T$ and $\vartheta_{0}$ by a precise numerical method and eq. (30), as shown in Figure 5. It is worth noting that when $\vartheta_{0} \approx \vartheta_{03}$, $T$ approaches infinity and when $\vartheta_{0}=60^{\circ}, T$ takes a very flat minimum (about 220.7 years).

\subsection{Other examples in the HS region and the TP region}

Below we choose three kinds of motion with the initial
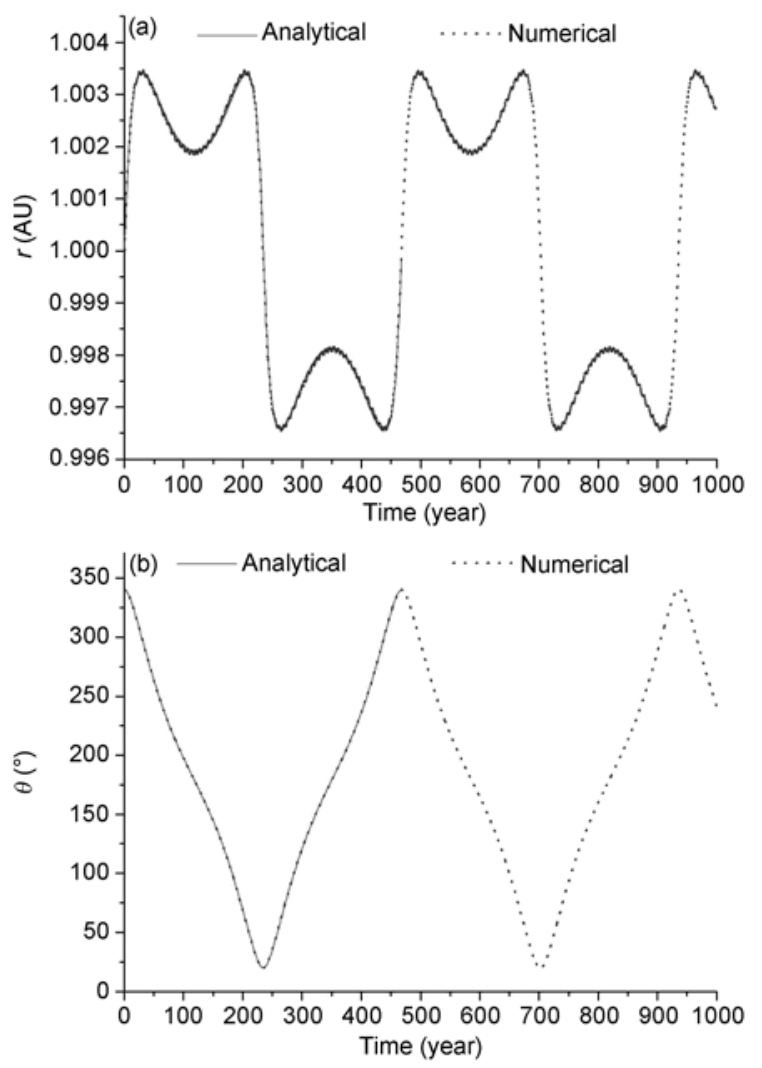

Figure 4 (a) The comparison of the results of $r$ respectively by the semi-analytical method and numerical integration; (b) the comparison of the results of $\vartheta$ respectively by the semi-analytical method and numerical integration.

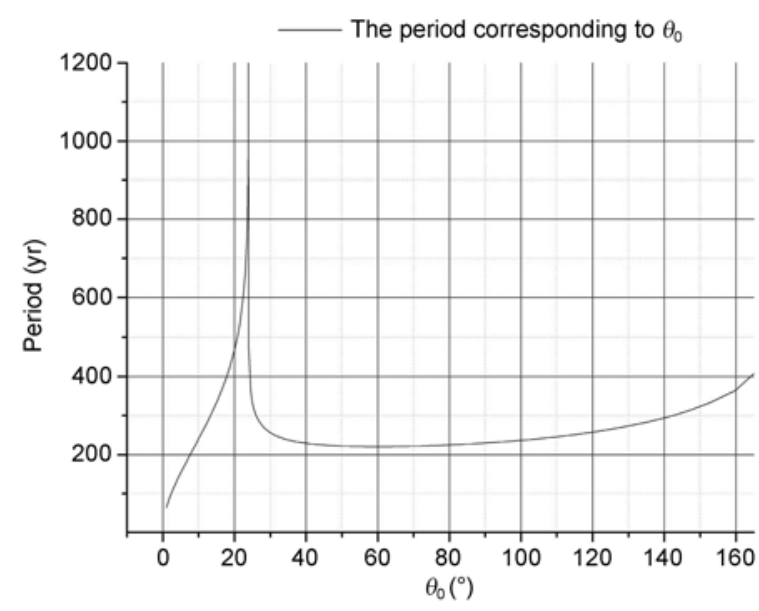

Figure 5 The relation between the period $T$ and $\vartheta_{0}$.

values to be $\vartheta_{0}=21^{\circ}$ in the HS region, and $\vartheta_{0}=30^{\circ}$ and $70^{\circ}$ in the TP region, respectively. For each kind we made a comparison of the results which were calculated by the semi-analytical method with those obtained by numerical integration, expecting further knowledge of the motion. Figure 6(a) describes the comparison of the results of $r$ from the semi-analytical method with those from numerical 

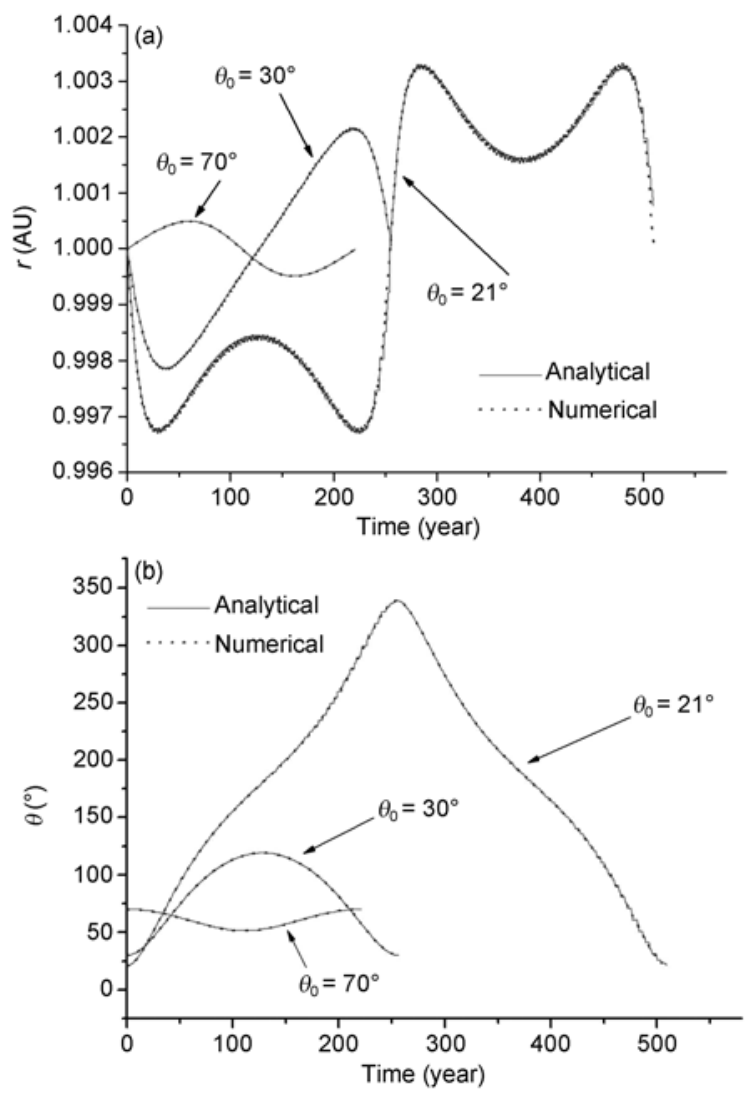

Figure 6 (a) $\vartheta_{0}=21^{\circ}, 30^{\circ}, 70^{\circ}$, the comparison; of the results of $r$ by the semi-analytical method with those by numerical integration; (b) $\vartheta_{0}=21^{\circ}, 30^{\circ}, 70^{\circ}$, the comparison of the results of $\vartheta$ by the semianalytical method with those by numerical integration.

integration for $\vartheta_{0}=21^{\circ}, 30^{\circ}, 70^{\circ}$ each; Figure $6(\mathrm{~b})$ describes the comparison of the results of $\vartheta$ from the semi-analytical method with those from numerical integration for $\vartheta_{0}=21^{\circ}, 30^{\circ}, 70^{\circ}$ each. The comparisons indicate that the extent of agreement is not only satisfactory, it is excellent for both research and application.

\section{Outlook}

In this paper we have presented an approximate semi-analytical method of the first order of the co-orbital restricted three-bodies problem, have made comparisons of the results of the calculation for initial values in the HS and TP regions (covering most of the range of the initial values), with the results from numerical integration, they agrees well with each other over the timescale of 1000 years, which is of certain significance to theoretical research and practical application. Further work involves, on the one hand, to conduct a deep theoretical investigation, on the other hand, to enlarge its application area. It includes the study of the stability of this kind of orbit, and the effect of orbital eccentricity of planets; the exploration of a more accurate analytical solution; the application to the study of the orbital evolution of the Trojan asteroids; the application to the study of the orbits of the near-earth asteroids; the application to the study of the orbit design of spacecraft being approximately co-orbital; and the investigation of the motion of co-orbital stellar families in the Milky Way galaxy.

The authors bear thanks towards Prof. Karsten Danzmann, from Max-Planck-Institut für Gravitationsphysik (Albert Einstein Institute), Yuehua Ma, and Wei-Tou Ni, both researchers from Purple Mountain Observatory, for their concern and support to this work and acknowledge financial support by the National Natural Science Foundation of China (Grant No. 10503013) and the Foundation of Minor Planets of Purple Mountain Observatory.

1 Hirayama K. Groups of asteroids probably of common origin. Astron J, 1918, 31: 185-188

2 Lemaitre A. Asteroid family classification from very large catalogues. In: Proceedings of the International Astronomical Union, 2004, IAUC197: 135-144. Cambridge: Cambridge University Press, 2005

3 Yi Z, Li G, Heinzel G, et al, Coorbital restricted problem and its application in the design of the orbits of the LISA spacecraft. Int J Mod Phys D, 2008, 17: 1005-1020

4 Li G, Yi Z, Heinzel G, et al, Methods for orbit optimization for the LISA gravitational wave observatory. Int J Mod Phys D, 2008, 17: 1021-1042

5 Mikkola S, Innanen K, Wiegert P. Stability limits for the quasi-satellite orbit. Mon Not Roy Astron Soc, 2006, 369: 15-24 